\title{
A Genetic Algorithm Approach for Optimal Distribution System Network Reconfiguration
}

\author{
Ganiyu Adedayo Ajenikoko, Adebayo Wasiu Eboda, Tunde Samuel Adeyemi \\ Department of Electronic and Electrical Engineering, Ladoke Akintola University of Technology, P.M.B 4000, Ogbomoso, \\ Nigeria. \\ Corresponding Email: ajeedollar@gmail.com
}

Received: 25.11.2017 Accepted:30.12.2017

\begin{abstract}
Electrical energy is an essential ingredient for the industrial and all-round development of any country. Power distribution systems are radial in configuration and this makes the networks hard to manage, thus, the need for optimization. This paper presents the optimization of network reconfiguration of distribution system using genetic algorithm to get the optimal switching scheme for network reconfiguration with objective function to reduce power loss and improve active power of the system. Load flow for the network reconfiguration problem was formulated as single objective optimization problem. The optimization model was simulated using MATLAB/SIMULINK and validated on standard IEEE 13-bus and 25-bus distribution test feeders. The result shows that active power increases by $91.1 \%$ (1.6469p.u.) while the power loss reduced by $99.4 \%$ (1.6372p.u.) for 13-bus system. For 25-bus system, active power increased by $27 \%$ (0.9154p.u.) and power loss reduced by $96.2 \%$ (4.3074p.u.) after optimization. The results provide solutions to the power distribution system for the optimal switching scheme for network reconfiguration with improvement in active power of the system. Total real power loss was minimized according to the corresponding fitness values of the genetic algorithm solutions. The paper provides technical information that could help in the future expansion and operation planning of the power distribution network.
\end{abstract}

Keywords: Distribution System, Load Flow, Network Reconfiguration, Distribution Test Feeder, Active Power, Power Loss, Genetic Algorithm.

\section{Introduction}

The quality of life in any country is highly dependent on a reliable electricity supply. The problems faced by electric power utilities in developing countries today is that the power demand is increasing rapidly whereas, the supply growth is constrained by aging generating and distributing assets, scarce resources for constructing new ones and other societal issues (Anoop, Yogendra, Manisha. 2011; Anthony, Ojo, Joseph. 2013).Ability to use electrical energy when required is one of the fundamental presumptions of a modern society and the introduction of sensitive machines into the network had increased the need for high reliability of supply. Deregulation and competition are forcing improvements in efficiency and reductions in cost while customers are becoming more sensitive to electrical disturbances and are demanding higher levels of service reliability (Olugbaga, Abdul-Ganiyu, Philips. 2013; Romeu. 2009).

Additional power generations that are adopted as measures to respond to the increasing power demand further strain the available distribution network links. It is therefore becoming essential to upgrade the existing network with optimized solutions or expand it with new lines to improve the reliability of the system while respecting the design criteria and assuring sustainable energy supply far into the future. The electric power distribution system is a robust network whose optimization requires lots of problem formulation. This is a very huge task for the distribution system operators and engineers (Suilaima, Nasir, Shamsudin, Sulaiman, Dahalan. 2015). Operation and planning of electric power systems involve a long list of challenging activities, many of which are directly or indirectly related with the optimization of certain objective functions. The goal of optimization of an electric power transmission system is to minimize objective functions subject to operational constraints of the power system (Maju, Leena, Sexena. 2016; Ogbogu, Anaemeje. 2011).

The dimension topology of distribution network has become larger due to increased scale of demand for the energy and this has contributed to the line losses significantly. However, many techniques have been identified in order to 
secure the planning due to the connection between the distribution network and consumers facilities. One of the techniques that have been applied to reduce the losses in large distribution network is reconfiguration system (Claudius, Awosope. 2014; Maju, Leena, Sexena. 2016). Reconfiguration of the distribution network systems are meant for power losses mitigation. During normal operation of a distribution network, the energy flow has a radial path and passes the normally closed switches. The network topologies in the distribution network reconfiguration change through the on/off of the sectionalizes and tie switches in order to get the optimal solution of the power losses (Alao, Amoo.2014; Sanni, Airoboman, Adagbe, amaize. 2011).

Therefore, the objectives of the work are to formulate a distribution load flow equations for reconfiguration of the distribution network, simulate the results using MATLAB/SIMULINK. Then optimized the simulation results using Genetic Algorithm for loss minimization and validate the optimization results on standard IEEE 13-bus and 25-bus distribution test feeder.

\subsection{Review of Related Works}

Researchers have used different optimization approaches for network reconfiguration in the past to find the most suitable configuration which consists of switches that will contribute in minimizing the power losses as well as to improve active power of the system. [6]presented a distribution network reconfiguration based on bacterial foraging optimization algorithm (BFOA) along with backward-forward sweep (BFS) load flow method and geographical information system (GIS) aimed at finding the radial structure that minimize network power loss while satisfying all operating constraints. Simulation was performed on the 33-bus system and the results were compared with other approaches.

Rama, Sivangaraju. (2010) analyzed network reconfiguration of distribution system using plant growth simulation algorithm with a view to enhancing speed and robustness of the system. The method was tested to reconfigure 69-node radial distribution system for loss minimization and load balancing. Eze, Agwu, Uzoechi. (2016) presented a branch exchange method and derived a simple formula to estimate the loss reduction with reconfiguration algorithms based on the heuristic optimization techniques as realistic and powerful solution schemes to obtain the global or quasi global optimal. Oni. (2015) discussed the application of modified form of particle swarm optimization as an optimization technique to the reconfiguration of electric distribution systems. The work used a novel strategy for the reconfiguration of a distribution system through the use of an optimized reconfiguration and multi-objective particle swarm optimization (MOPSO). However, the algorithm did not perform well in terms of better convergence time.

\subsection{Electrical Distribution System}

Distribution system is the final stage in the delivery of electric power. It carries electricity from transmission system to individual consumers. The systems are designed radially for effective coordination between feeder's protection systems and to lower the short circuit currents. The distribution feeders have two types of switches: normally closed (sectionalizing) switches and normally opened (tie) switches (Oluwole, Babriel, Akinwale. 2014). The major concern for distribution utilities is to reduce the power losses in distribution network. Failure statistics reveal that the electrical distribution systems constitute the greatest risk to the uninterrupted supply of power. However, distribution systems have received less attention than the generation and transmission parts of the overall electrical power systems (Ikeli. 2009).

Distribution systems are less capital-intensive and their failures cause more localized effects compared to generation and transmission systems. Electrical power systems operation must be able to generate sufficient power for the present and future consumption and transmit electric power to load centers and distribute same in a safe and efficient manner to facilitate the final utilization (Subramanian, Jaisina, Swathana, Neelan. 2015). Distribution networks are built as interconnected networks while in operation they are arranged in a radial tree structure. This means that a distribution system is divided into subsystems of radial feeders, which contain a number of normally closed (NC) switches and normally opened (NO) switches. Distribution substations are connected to the transmission system and lower the transmission voltage to medium voltage with the use of transformers (Rama, Sivangaraju. 2010; Ikeli. 2009).

The typical radial distribution network depicted in Figure 1 is such that the feeder section forms a set of trees where each sink node is supplied from exactly one source node. Power is supplied from the secondary substations through feeders at $11 \mathrm{KV}$ to distribution substations. Power is fed to the different consumers through a system of conductors. The most common system of secondary distribution is $415 / 230 \mathrm{~V}$, 3phase 4-wire system. The single-phase residential lighting load is connected across single-phase lines directly. Most residential consumers use single-phase meters. Industrial and large commercial customers take their supply directly from 11 $\mathrm{kV}$ and use 3-phase meters (Suilaima, Nasir, Shamsudin, Sulaiman, Dahalan. 2015). 


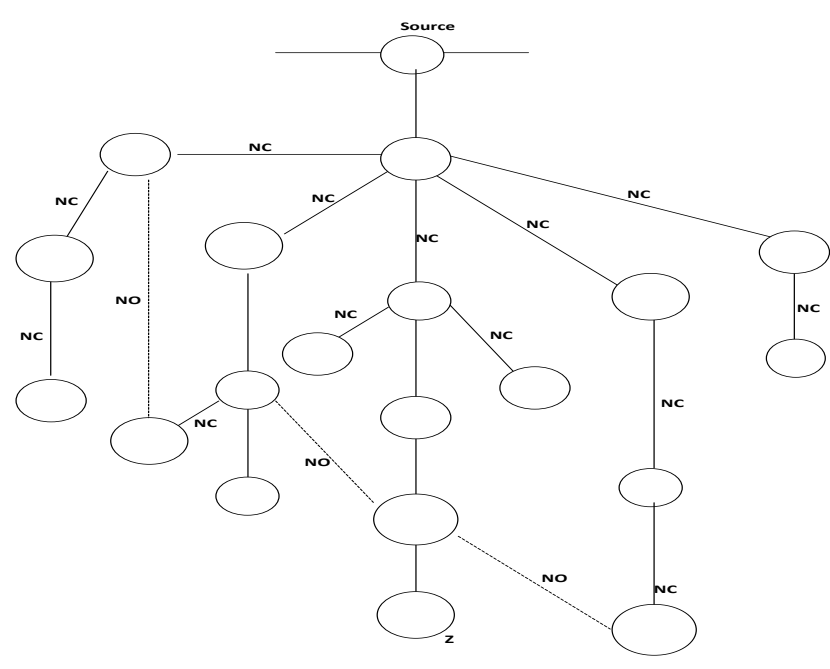

Figure 1 Typical radial distribution system

\subsection{Network Reconfiguration in Electrical Distribution System}

Network reconfiguration is the process of operating switches to change the circuit topology so that operating costs are reduced while satisfying the specified constraints (Anoop, Yogendra, Manisha. 2011; Anthony, Ojo, Joseph. 2013). Distribution network planning requires feeder reconfiguration on a seasonal and annual basis with the potential to provide required benefit, since the load varies continuously as the network expands (Maju, Leena, Sexena. 2016). This potential can be exploited by distribution management system where the switches can be opened and closed on a real-time basis. The concept of reconfiguration of radial distribution network is aimed at improving the system performance under different operating conditions. Distribution network reconfiguration (DNR) is the most economical method to reduce the power losses in distribution network (Alao, Amoo.2014). Optimal distribution planning involves network reconfiguration for distribution loss minimization, load balancing under normal operating conditions and fast service restoration, minimizing the zones without power under failure conditions (Anoop, Yogendra, Manisha. 2011).

These switching are performed in such a way that the radiality of the network is maintained and all the loads are energized. A normally opened tie switch is closed to transfer a load from one feeder to another while an appropriate sectionalizing switch is opened to restore the radial structure. The benefits of feeder reconfiguration include (Eze, Agwu, Uzoechi. 2016; Anthony, Ojo, Joseph. 2013):

i. Restoring power to any outage partitions of a feeder.

ii. Relieving overloads on feeders by shifting the load in real time to adjacent feeders.

iii. Reducing resistive line losses.

\section{Materials and Methods}

In this paper, distribution load flow branch equations of a set of recursive equations was used for reconfiguration of the distribution network. This uses the active power and voltage at the sending end of a branch to express the same quantities at the receiving end of the branch. In order to study the net effects of network reconfiguration in a distribution power system by changing the switching states of normally closed (sectionalizing) switches and normally opened (tie) switches, script codes were written in MATLAB and simulations was carried out on standard IEEE 13-bus and 25bus distribution test feeder. The total active power and system losses were calculated by distributed power flow analysis and tabulated. Genetic Algorithm Optimization for power loss minimization approach was implemented for optimal reconfiguration of the system.

\subsection{Problem Formulation}

The problem of distributed network reconfiguration is to find best configuration of radial network having minimum power losses. Since many switching combinations are possible in a distribution network, finding the best configuration is a complex constrained optimization problem. The mathematical formulations for objective function for power loss minimization are as follows:

Minimize $f_{U \in S}(x, u)$

Such that $\mid f(x, u)=0$

Where

$$
\mid f(x, u) \leq 0
$$

S is the set of all possible network configurations and F and G arenon-linear functions.

Moreover, there are some constraints considered during the process of analysis. The constraints are:

i. The voltage constraints: To maintain the power quality of the system, the voltage magnitude should be based on within 1.05p.u. and 0.95p.u. $V_{\text {Min }} \leq V_{\text {Bus }} \leq V_{\text {Max }}$

ii. Power flow constraints: Each and every branch in the power flow has its own permissible range. This range should be followed clearly and the constraint strictly lies within it.

iii. Radial configuration constraints: This constraint should be considered to avoid any excess of current flow through the system.

\subsection{Distribution Load Flow Calculation Method}


In order to evaluate the objective function of the problem, load flow equation was developed by calculating the voltage at each bus, beginning from the generator bus to the load buses using the currents calculated in backward sweep method.

Assumptions used for load flow equations:

i. The system is a balanced 3-phase system

ii. Shunt capacitor was placed at the nodes of the system as reactive power injections.

iii. Number of the switches was increased for radial configuration format.

Consider a simple radial distribution network shown in Figure 2.

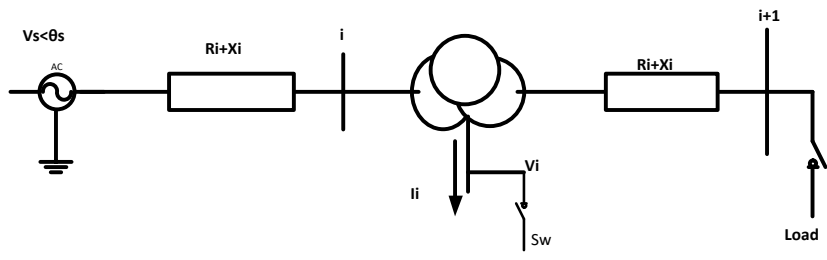

Figure 2 Single line diagram of distribution network

From the line diagram, the complex power at bus $i$ is

$$
\begin{aligned}
& S_{i}=V_{i} I_{i}^{*}=P_{i}+j Q_{i} \\
& I_{i}=\frac{\sqrt{P_{i}{ }^{2}+Q_{i}{ }^{2}}<\tan ^{-1}\left(-Q_{i} / P_{i}\right)}{\left|V_{i}\right|<-\theta_{V_{i}}} \\
& I_{i}=\left|I_{i}\right|=\frac{\left(P_{i}{ }^{2}+Q_{i}{ }^{2}\right)^{\frac{1}{2}}}{\left|V_{i}\right|}
\end{aligned}
$$

Voltage at receiving end of bus $(i+1)$ is given by:

$$
V_{i+1}^{2}=V_{i}^{2}+\left(R_{i}^{2}+X_{i}^{2}\right) \frac{I_{i}}{V_{i}}-2\left(R_{i} P_{i}+X_{i} Q_{i}\right)
$$

The active and reactive power at receiving end at bus $(i+1)$ is given by:

$$
\begin{gathered}
P_{i+1}=P_{i}-P_{L i}-R_{i} \frac{I_{i}}{V_{i}} \\
Q_{i+1}=Q_{i}-Q_{L i}-X_{i} \frac{I_{i}}{V_{i}}
\end{gathered}
$$

The real and reactive power flow in the line between $(i+1)$ and nth buses are:

$$
\begin{gathered}
P_{\text {Loss }}=\sum_{i=1}^{n_{b r}}\left|I_{i}\right|^{2} r_{i} \\
Q_{\text {Loss }}=\sum_{i=1}^{n_{b r}}\left|I_{i}\right|^{2} x_{i}
\end{gathered}
$$

Nevertheless, reactive power loss is not less important due to the fact that reactive power flow in the system needs to be maintained to a certain amount for sufficient voltage level.

where

$S_{i}$ : Complex power at bus $i$

$P_{i}$ : Active Power at bus $i$

$Q_{i}$ : Reactive Power at bus $i$

$V_{i}$ : Sending voltage at bus $i$

$I_{i}$ : Sending end current at bus $i$

$\left|V_{i}\right|$ : Voltage magnitude at bus $i$

$\left|I_{i}\right|$ : Current magnitude at bus $i$

$\theta_{V_{i}}$ : Voltage angle at bus $i$

$\theta_{i}$ : Load current angle at bus $i$

$V_{i+1}$ : Receiving end voltage at bus $(i+1)$

$R_{i+1}, X_{i+1}$ : Resistance and Reactance at bus $(i+1)$

$P_{i+1}, Q_{i+1}$ : Active and Reactive power at bus $(i+1)$

$P_{L i}, Q_{L i}$ : Active and Reactive power loss at bus $i$

$n_{b r}$ :Total number of branches in the distribution radial network,

\section{Simulation and Optimization for the Network Reconfiguration}

For the optimization of network reconfiguration, the distribution load flow equation for network reconfiguration was simulated according to the load flow algorithm. Genetic algorithm (GA) was employed for the minimization of power loss and power improvement and to place the switch in an appropriate location. The simulation was carried out according to the algorithm below and the flowchart is depicted in Figure 3.

Step 1: Read the system data

Step 2: Calculate the system branches current

Step 3: Calculate the system active and reactive

power

Step 4: Update the bus voltage using branch current.

Step 5: If the difference between the iterationsis more than preset value (1.0 p.u), then go to step 2, else calculate the power losses of the system.

Step 6: Select possible location based on areas that have large losses.

Step 7: For each selected location, recalculate system losses.

Step 8: If the system losses areacceptable, stop and rank each location according to the losses, else reject, then go to step6. 
From the flowchart, 13-bus and 25-bus test data was inputted for simulation and distribution load flow algorithm was applied for system losses calculation. GA was then employed for the minimization of the system losses by applied a selection to the current population of the distribution network to create an intermediate distribution population.

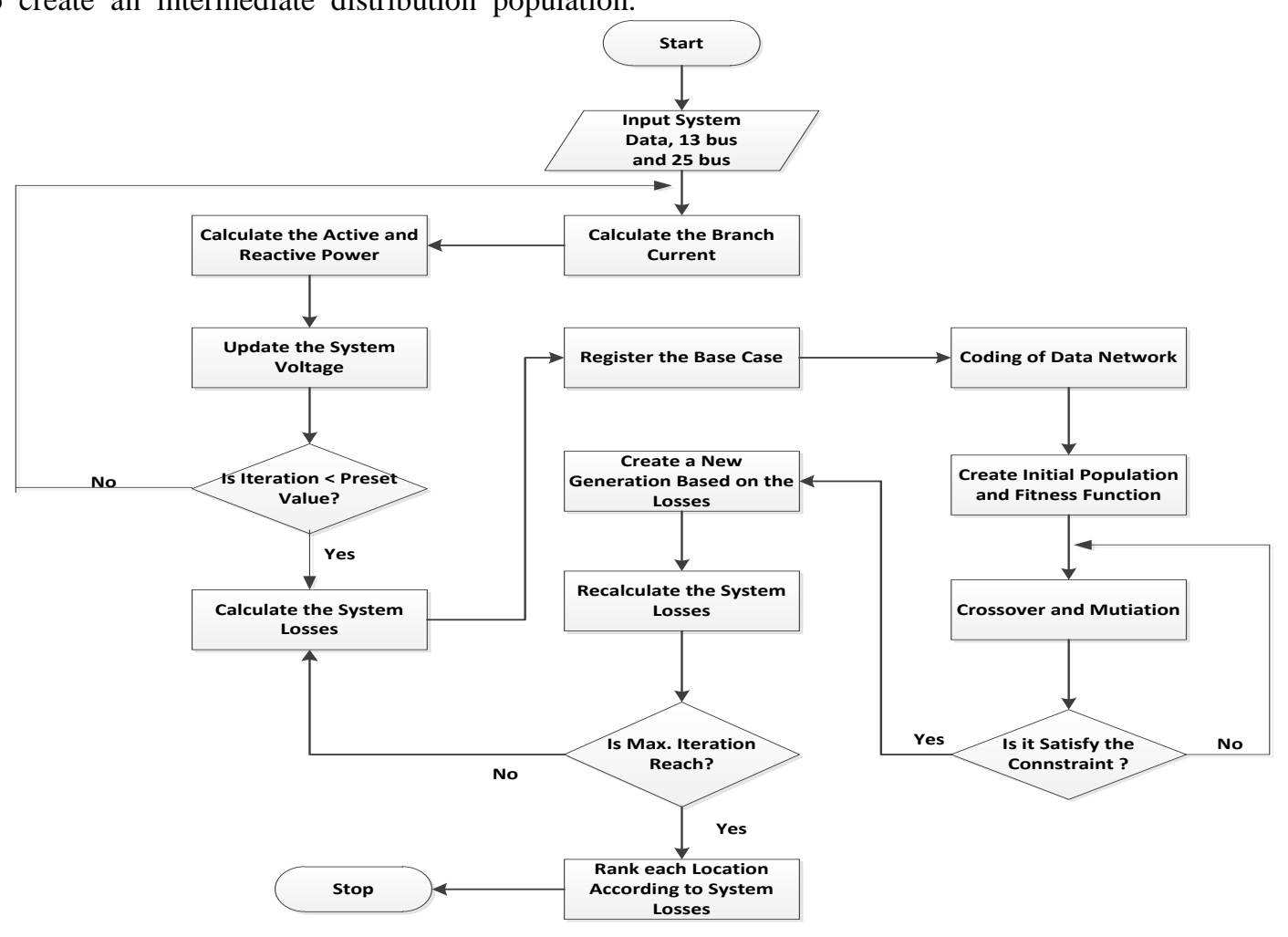

Base on the string's rank in the population, the recombination and mutation are then applied to the intermediate population of the network to create the next distribution network population. This process constitutes one generation in the execution of the Genetic Algorithm.

Figure 3 Flowchart for the optimization of network reconfiguration

\section{Discussion of Results}

The optimized distribution load flow algorithm was tested on standard IEEE 13-bus and 25-bus test feeder. The test feeders are reconfigured as line number. The corresponding results for two test feeders are presented according to active power and system loss. The simulationsresultsare presented in Figures 4 to 13.

Figure 4 shows how the active power varies with the line number for 13-bus test feeder without reconfigurationwith a pre-voltage of $4.16 \mathrm{kV}$ and base MVA value of 25 . Lines $1,2,3$ and 4have active power values of 0.1101, 0.0092, 0.0531 and 0.0678p.u. respectively. Lines 5, 6,7 and 8 have active power values of $0.0867,0.0343,0.0565$ and 0.0781 p.u. respectively. Lines $9,10,11$ and 12 recorded an increase in active power values of 0.0778, 0.0737, 0.0458 and 0.0545 Sp.u respectively as a result of the loadings of the feeders. Figure 5 indicates the variation of power loss with line numbers for 13-bus test feeder without reconfiguration. Lines 1, 2, 3 and 4 recorded power loss values of 0.1101 , $0.1476,0.104$ and 0.1486p.u. respectively. Power losses at lines 5, 6, 7 and 8 are 0.1610, 0.1053, 0.1443 and 0.1456p.u. respectively. Lines 9, 10, 11 and 12recorded an increase in power losses of $0.1501,0.1421,0.1401$ and 0.1481 p.u. respectively due to resistance value at each bus.

\section{Figure}

illustratesthe optimal active power for 13-bus test feeder after network reconfiguration. Lines $1,2,3$ and 4 have active powers of $0.1150,0.1478,0.1401$ and 0.1469 p.u. respectively. Lines 5, 6, 7 and 8 have values of optimal active power of 0.1606, 0.1098, 0.1405 and 0.1429p.u. respectively. Lines 9, 10, 11 and 12 have optimal active power values of $0.1508,0.0737,0.0458$ and 0.0545 p.u. respectively indicating an increase in active power afterreconfiguration due to active load power at each bus as well as the optimization parameters of active load power at each bus.

Figure 7 shows the variation of optimal power loss of 13-bus test feeder with line numbers after network reconfiguration. System loss with optimization for lines 1, 2, 
3 and 4 are 0.0023, 0.0021, 0.0014 and 0.0017p.u. respectively. Lines 5, 6, 7 and 8 have the values of optimal power losses of $0.0002,0.0003,0.0004,0.0005$ p.u respectively. Lines 9, 10, 11 and 12 have values of power losses $0,0.0005,0$ and 0.0003p.u respectively. Lines 9 and 11 appeared to have no power loss for all the buses because they are voltage-controlled buses.

The results show that the total active powers without network reconfiguration were0.7476p.u and 1.4284p.u. after optimization with network reconfiguration. The active power increases by 0.6808 p.u.(which is $91.1 \%$ increase).The total power losses without network reconfigurationwere 1.6469p.u. and 0.0097p.u. after optimization with network reconfiguration. Power losses reduced by $1.6372 p . u$, that is, a $99.4 \%$ reduction. The result indicates an increase in active power and loss reductionin the system after network reconfiguration. This is due to the high resistance of load data at each feeder.

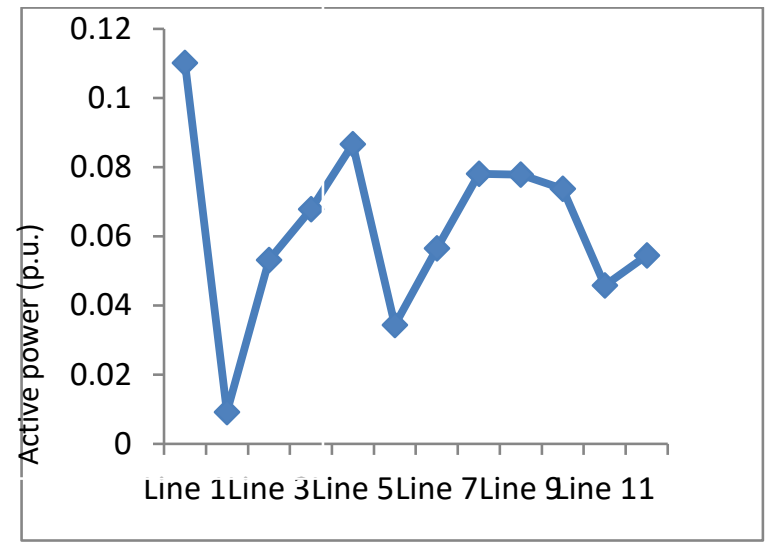

Figure 4 Active power without network reconfiguration versus line number

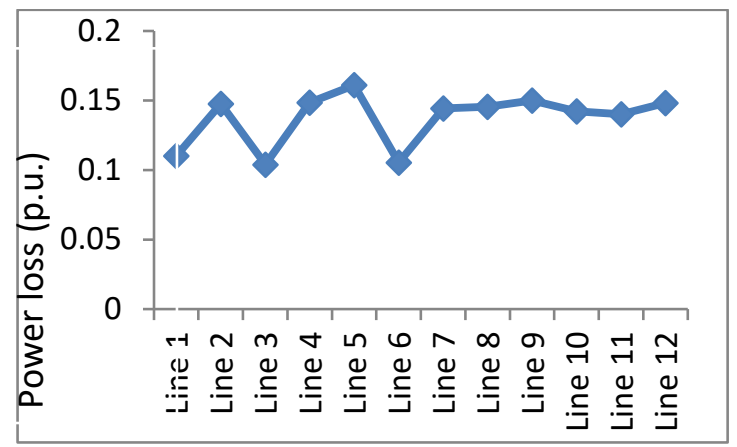

Figure 5 Power Loss without network reconfiguration versus line number

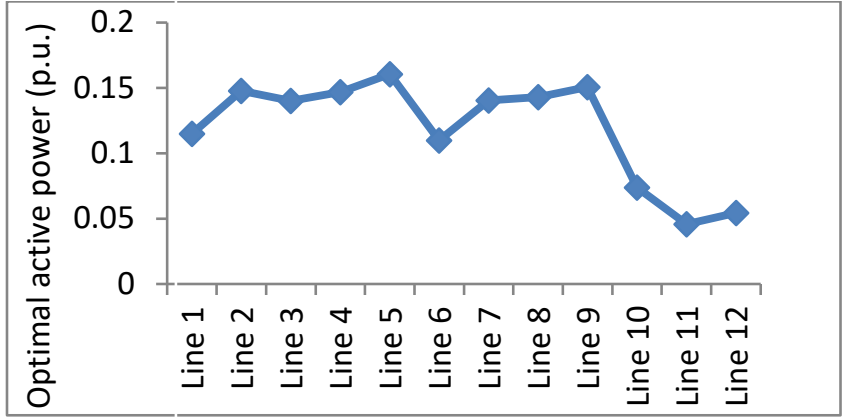

Figure 6 Optimal active power with network reconfiguration versus line number

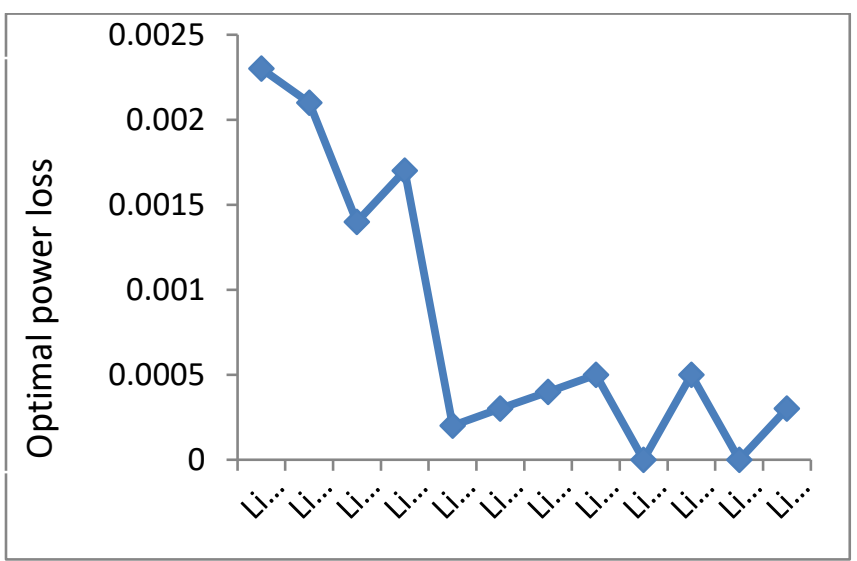

Figure 7 Optimal power loss with network reconfiguration versus line number

Figure 8 shows the variation of active power with the line numbersfor 25-bus test feeder without network reconfiguration with a pre-voltage of $4.16 \mathrm{kV}$ and base MVA value of 35. The active power for lines 1, 2, 3, 4, 5 and 6 are $0.1582,0.1138,0.1252,0.1292,0.1531$ and 0.1383p.u. respectively. Lines 7, 8, 9, 10, 11 and 12 have values of active power of $0.1165,0.1531,0.1523,0.1307,0.1243$ and 0.1512 p.u. respectively. Active power at lines $13,14,15,16$, 17 and 18 are $0.1489,0.1511,0.1512,0.1512,0.1512$ and 0.1338p.u respectively. Active power for lines 19, 20, 21, 22, 23 and 24 are $0.1550,0.1201,0.1550,0.1138,0.1432$ and 0.1432p.u. respectively. The variation between power losses for 25-bus test feeder with line number without network reconfiguration is depicted in Figure 9. The power losses for lines 1, 2, 3, 4, 5 and 6 are 0.1510, 0.1576, $0.1710,0.1701,0.2154$ and $0.1003 p . u$ respectively. Lines 7 , $8,9,10,11$ and 12 have the values of power losses of 0.1302 , $0.2154,0.2154,0.2041,0.1854$ and 0.2154 p.u. respectively. Lines 13,14, 15, 16, 17 and 18 have the values of power losses of $0.2123,0.2154,0.2154,0.2143,0.2153$ and 0.1810p.u respectively. Power losses for lines 19, 20, 21, 22, 23 and 24 are $0.2134,0.2134,0.1142,0.1421,0.2101$ and 0,2007p.u. respectively. 
The variation of the optimal active power for 25-bus test feeder with line numbers after network reconfiguration is illustrated in Figure 10. Lines 1, 2, 3, 4, 5 and 6 have values of optimal active power of $0.1582,0.1578,0.1782,0.1732$, 0.2157 and 0.1105 p.u. respectively. The optimal active power for lines 7, 8, 9, 10, 11 and 12 are 0.1601, 0.2157, 0.1531, 0.1383, 0.1265 and 0.1531 p.u respectively. Lines $13,14,15$, 16,17 and 18 have values of optimal active power of 0.2157 , 0.2052 , 0.1837, 0.2157,0.2176 and 0.2157p.u. respectively. Optimal active power for lines 19, 20, 21, 22, 23 and 24 are $0.2157,0.1870,0.2117,0.1146,0.1454$ and 0.2103 p.u. respectively.

Figure 11 illustrates the correspondence between optimal power loss and line numbers for 25-bus test feeder after network reconfiguration. The optimal power losses for lines 1, 2, 3, 4, 5 and 6 are 0.0096, $0.0159,0.0197,0.0187,0.0039$ and 0.0186 p.u respectively. Lines 7, 8, 9, 10, 11 and 12 have values of optimal power losses to be $0.0039,0.0187,0,0,0.0112$ and 0.0129 p.u. respectively. The optimal power lossesfor lines 13, 14, 15, 16, 17 and 18 are $0,0,0,0,0.0079$ and 0 p.u respectively.Optimal power loss for lines 19, 20, 21, 22, 23 and 24 are 0, 0.0117, 0, $0.0198,0$ and 0 p.u. respectively. The optimal power losses for lines $21,22,23$ and 24 are $0,0.0189,0$ and 0 p.u. respectively. Lines 9, 10, 12, 13, 14, 15, 16, 17, 19, 21, 23 and 24 appeared to have no power losses out of all the lines because they are voltage-controlled buses.

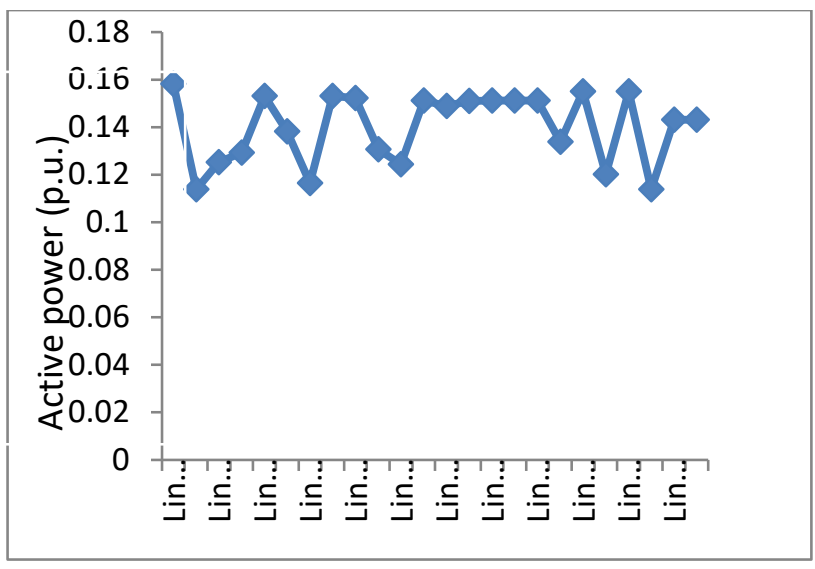

Figure 8 Active Power without network reconfiguration versus line number

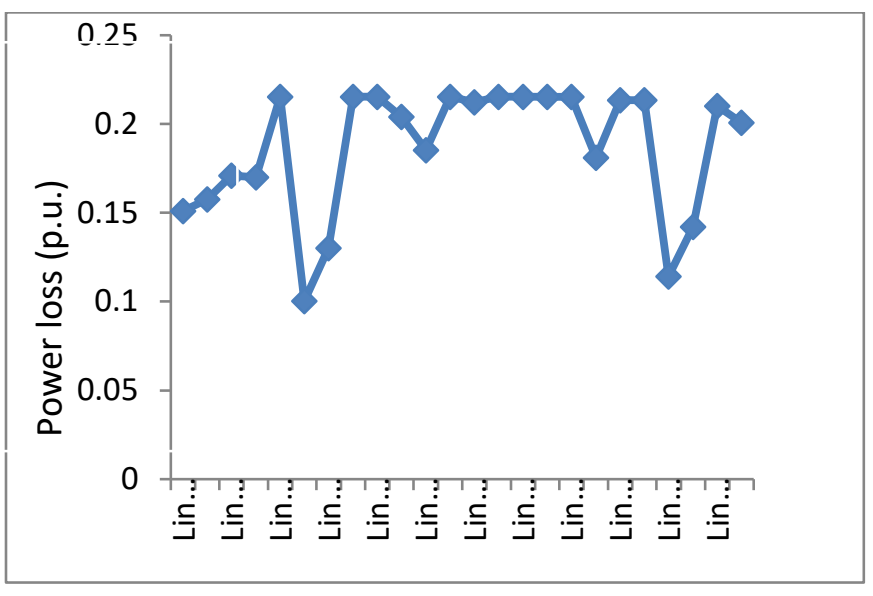

Figure 9 Power Loss without network reconfiguration versus line number

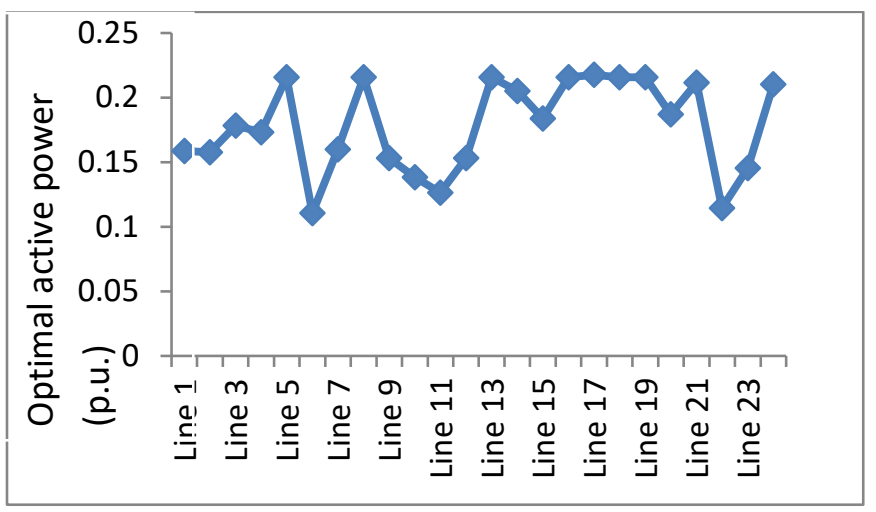

Figure 10 Optimal active power with network reconfiguration versus line number

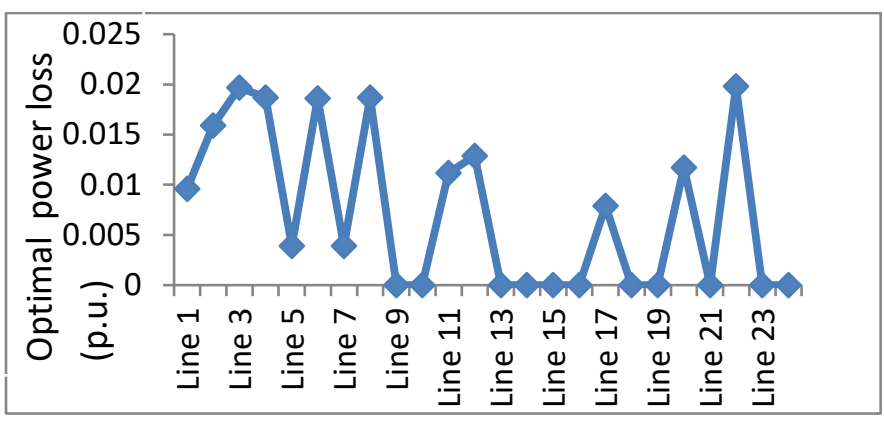

Figure 11 Optimal power loss with network reconfiguration versus line number

The result for 25-bus test feeder reveals that the total active power without network reconfiguration was3.3636 p.u and 4.279 p.u. after optimization with network reconfiguration. The active power increases by 0.9154 p.u. (which is $27 \%$ increment). The totalpower loss without network reconfiguration is 4.4799 p.u.and 0.1725 p.u. after optimization with network reconfiguration. Power losses reduced by 4.3074 p.u, that is, a $96.2 \%$ reduction. The result 
reveals an increase in active power and reduction in system loss after network reconfiguration. This is due to the load data at each feeder indicating a reduction in power losses.

\section{Conclusion}

Network reconfiguration of the distribution system for active power improvement and system loss has been presented in this paper. Genetic algorithm was employed for optimization to address the power loss minimization and active power improvement. The results indicate that active power increases by $91.1 \%$ (1.6469 p.u.) while the power loss reduced by $99.4 \%$ (1.6372 p.u.) for 13-bus test feeder while for 25-bus test feeders, active power increase by $27 \%$ (0.9154 p.u.) and power loss reduced by $96.2 \%$ (4.3074 p.u.) after optimization.The results from the two test feeder demonstrated the applicability of the method, thus the result will help in developing a multi objective based reconfiguration model for distribution network to obtain optimal switching state. The paper will form a basis for initial technical information that could help in the future expansion and operation planning of the distribution network.

\section{Conflicts of interest}

The authors have no conflicts of interest to declare.

\section{References}

[1] Anoop A, Yogendra K, Manisha D. Reconfiguration of electric distribution network using modified particle swarm optimization. International Journal of Computer Application. 2011; 34(6):54-62.

[2] Anthony U.A, Ojo A.A, Joseph C.E. Asset maintenance planning in electric power distribution network using statistical analysis of outage data. Electrical Power and Energy Systems. 2013; 47(6):424-435.

[3] Olugbenga T.K, Abdul-Ganiyu A.J, Phillips D. A. The current and future challenges of electricity market in Nigeria in the face of deregulation process. African Journal of Engineering Research. 2013; 1(2):33-39.

[4] Romeu M.V. Network reconfiguration to improve reliability and efficiency in distribution systems. INESC Coimbra - Jornadas. 2009; 4(5):1-13.

[5] Sulaima M.F, Nasir M.N, Shamsudin N.H, Sulaiman M, Dahalan W.M. Implementation of modified EPSO technique in $69 \mathrm{kV}$ distribution network reconfiguration for losses reduction. International Journal of Engineering and Technology. 2015; 7(2):502-509.

[6] Manju M.A, Leena G, Saxena N.S. Distribution network reconfiguration for power loss minimization using bacterial foraging optimization algorithm.
International Journal of Engineering and Manufacturing. 2016; 2(3):18-32.

Ogbogu N.O, Anaemeje G. Electric power transmission optimization. JORIND. 2011; 9(1):1-9.

Claudius A, Awosope C.A. Nigeria electricity industry: issues, challenges and solutions. Media and Corporate Affairs, Covenant University Press, Public Lecture Series. 2014; 3(2):1-40.

[9] Alao, A.A, Amoo, A.A. Enhancing the performance of electricity distribution companies in Nigeria via internal control system. Research Journal of Finance and Accounting. 2014; 5(22):197-214.

[10] Sanni T.F, Airoboman A, Adoghe A, Amaize P. A model for transmission grid decongestion. $3^{\text {rd }}$ International Conference on African Development Issues, Covenant University Press. 2011 (pp. 209292).

[11] Rama R.P, Sivanagaraju S. Radial distribution network reconfiguration for loss reduction and load balancing using plant growth simulation algorithm. International Journal on Electrical Engineering and Informatics. 2010; 2(4):266-277.

Eze C.U, Agwu D.D, Uzoechi L. O. A new proposed demand side management technique. International Journal of Engineering Sciences \& Emerging Technologies. 2016; 8(6):271-281.

[13] Oni J.O. Development and application of modified multi-regression algorithm for demand side load forecasting of electrical power. A Thesis submitted for the Award of the degree of Doctor of Philosophy in Electrical Engineering, University of Ilorin, Ilorin, Nigeria. 2005; pp. 1-160.

[14] Oluwole C.A, Babriel B.I, Akinwale M.O. Optimal transformer allocation in electrical distribution using genetic algorithm. International Journal of Physical Sciences. 2014; 9(14):309-319.

[15] Ikeli, H.N. A critical analysis of transient stability of electrical power system:a case study of Nigerian $330 \mathrm{kv}$ power system. A project work submitted in partial fulfillment of the requirement for the award of Master of Engineering Degree (M. Eng) in Electrical Engineering, University of Nigeria, Nsukka. 2009; pp. 1-214.

[16] Subramanian A, Jaisiva S, Swathana R, Neelan S. Optimal allocation of FACTS device for voltage profile enhancement. International Journal of Enhanced Research in Science, Technology \& Engineering. 2015; 4(8):187-193. 